\title{
Pregnancy Tumor
}

${ }^{1}$ Shwetha Chikkaboraiah, ${ }^{2}$ Rajiv Nidasale Puttaswamaiah, ${ }^{3}$ Sushama R Galgali

\section{ABSTRACT}

Aim: The aim of this case report is to present a case of rapidly growing large pregnancy tumor which did not regress even after parturition.

Introduction: Pyogenic granuloma is a commonly occurring benign inflammatory hyperplasia of the skin and oral mucosa. It usually arises in response to nonspecific infection as sessile or pedunculated, erythematous, exophytic papule or nodule with a smooth or lobulated surface that bleeds easily. Pyogenic granuloma occurring during the pregnancy is called as pregnancy tumor.

Case report: In this case report, a rapidly growing pregnancy tumor in a 23-year-old female is described and the clinical features, histopathologic features and management of the lesion are discussed. Pregnancy tumor rarely reaches $2 \mathrm{~cm}$ in size, however in our case the lesion had enlarged to an extent that it was interfering with occlusion $(3 \times 2 \mathrm{~cm})$. Even after parturition the enlargement showed no regression in size, so the lesion was surgically excised.

Conclusion: Lack of awareness among pregnant patients about oral health care during pregnancy results in occurrence of various oral lesions which challenges the dentist and gynecologist in providing proper treatment. Hence creating oral health care awareness among such patients is of utmost importance.

Clinical significance: The prenatal oral health check-up and counselling is important in preventing the occurrence of various oral lesions during pregnancy.

Keywords: Female sex hormones, Gingiva, Inflammatory hyperplasia, Lobular capillary hemangioma, Pregnancy tumor, Pyogenic granuloma.

How to cite this article: Chikkaboraiah S, Puttaswamaiah RN, Galgali SR. Pregnancy Tumor. J Health Sci Res 2016;7(1):23-27.

Source of support: Nil

Conflict of interest: None

\section{INTRODUCTION}

Pyogenic granuloma is a benign inflammatory lesion that mostly occurs on the gingiva of young females in puberty, females taking contraceptives and pregnant women. ${ }^{1}$

\footnotetext{
${ }^{1}$ Postgraduate Student, ${ }^{2}$ Reader, ${ }^{3}$ Professor and Head

${ }^{1-3}$ Department of Periodontics, Vokkaligara Sangha Dental College and Hospital, Bengaluru, Karnataka, India
}

Corresponding Author: Shwetha Chikkaboraiah, Postgraduate Student, Department of Periodontics, Vokkaligara Sangha Dental College and Hospital, Bengaluru, Karnataka, India Phone: +918026950662, e-mail: shwethac62@gmail.com
Hullihen SP in 1844 reported the first case of pyogenic granuloma which is probably the first case in the English literature. In 1897, two French surgeons, Poncet and Dor named this lesion as "Botryomycosis hominis." In 1904, Hartzell MB introduced the term "pyogenic granuloma" or "granuloma pyogenicum." ${ }^{2}$

The term pyogenic granuloma is a misnomer as it is not related to any infection, does not contain pus, and is not a true granuloma. It develops in up to $5 \%$ of pregnancies and hence, terms like "pregnancy tumor" and "granuloma gravidarum" are commonly used. ${ }^{3}$ The pregnancy tumor develops as a result of an exaggerated inflammatory response to local irritations, then enlarges rapidly, bleeds easily, and becomes hyperplastic and nodular. The tumor may be sessile or pedunculated and may range from purplish red to deep blue in color with small fibrin spots. Gingiva is the most common site involved (70\%) followed by tongue, lips, buccal mucosa, and the palate. ${ }^{4}$

Prominent endothelial cell proliferation and associated inflammation are its histopathological characteristics. It is generally believed that female sex hormones play important roles in its pathogenesis because of higher prevalence in females with high levels of sex hormones and regression of the lesions after child birth. Elevated sex hormones alone cannot cause the lesions since the lesions only occur on sites with local irritants and can be prevented by the removal of local irritants. ${ }^{1}$

This article describes a case of pregnancy tumor in a 23-year-old pregnant patient.

\section{CASE REPORT}

A 23-year-old pregnant woman reported to the Department of Periodontics, Vokkaligara Sangha Dental College and Hospital, Bengaluru with a chief complaint of bleeding gums while brushing and swelling on the gums in the upper left back tooth region since 1 month. The lesion was painless and asymptomatic except for the slight discomfort due to the gingival overgrowth. There was no difficulty in speech or mastication. The medical history revealed that the patient was in her 1st trimester of pregnancy.

On clinical examination a small confined soft tissue mass measuring $4 \times 3 \mathrm{~mm}$ was noticed interdentally between 24 and 25. The lesion was ovoid in shape, reddish pink in color, and the surface was smooth without any ulcerations. It extended from the distal line angle of 25 to the distal line angle of 24 . On palpation the swelling 
was soft in consistency, pedunculated, nontender and on provocation bled easily. Supragingival and subgingival calculus was also present. After the history and clinical examination the provisional diagnosis of pyogenic granuloma (pregnancy tumor) was made. Differential diagnosis of peripheral giant cell granuloma and peripheral ossifying fibroma was considered.

Initially, the lesion was treated by nonsurgical periodontal therapy. Instructions regarding maintenance of oral hygiene were given.

At 2 nd recall visit (5th month of pregnancy), it was observed that in spite of instituting non-surgical periodontal therapy and oral hygiene measures, the growth had increased in size (from $4 \times 3 \mathrm{~mm}$ to $3 \times 2 \mathrm{~cm}$ involving 24,25 , and 26) to an extent that it was interfering with the occlusion (Figs 1 and 2). The oral hygiene maintenance was poor even after educating the patient about various oral hygiene measures. The lesion was extending anterioposteriorly from mesial surface of 24 to distal surface of 26 and superioinferiorly from upper buccal vestibule to lower buccal vestibule, covering the entire buccal surfaces of $24,25,26,34,35$, and 36 . The growth was firm on palpation, nontender. Bleeding on provocation was positive. There was grade-I mobility in relation to 24 .

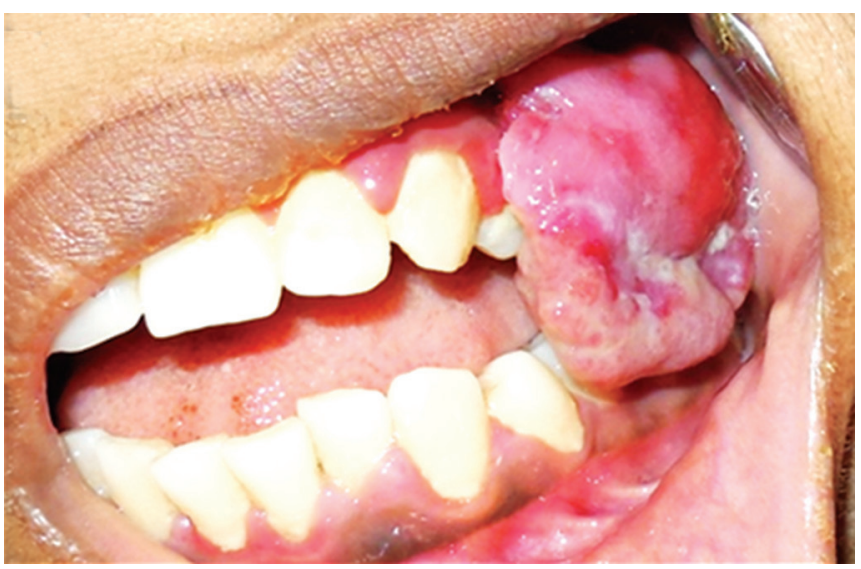

Fig. 1: Preoperative (lateral view)

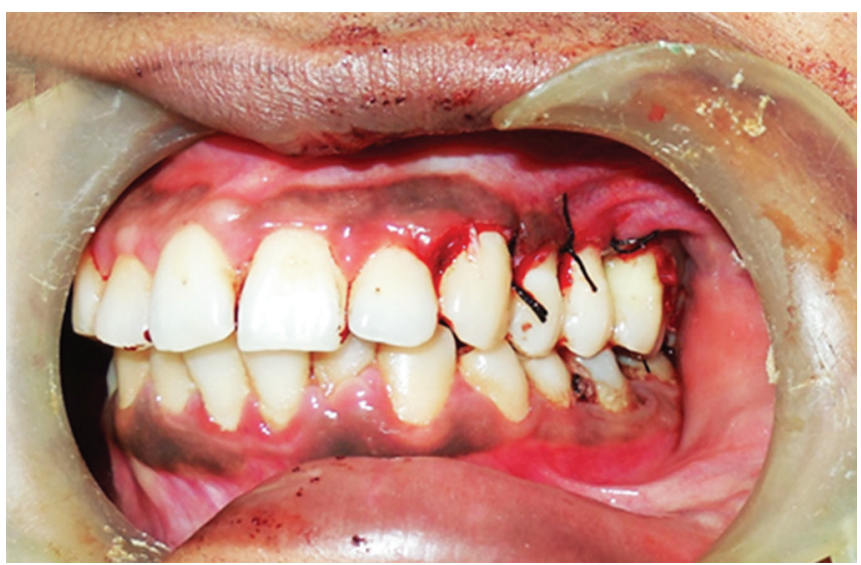

Fig. 3: After excision
Since the lesion was interfering with mastication, gynecologist's opinion was taken and surgical excision of the lesion was planned. The patient was apprehensive and did not give consent for the planned excision. So, the patient was advised to visit the department after parturition. Patient reported 2 months after uneventful parturition. The lesion had not regressed and remained the same $(3 \times 2 \mathrm{~cm})$. Thus it was excised with patient's consent under local anesthesia. Open flap debridement and gingival contouring were carried out simultaneously (Fig. 3). Sutures and periodontal dressing was given. The patient was discharged from the operatory after postsurgical instructions and recalled after 1 week for a follow-up. The excised lesion (Figs 4 and 5) was sent for histopathological examination.

Microscopic examination of hematoxylin and eosin (H\&E) stained sections (Figs 6 and 7) showed parakeratinized stratified squamous epithelium of irregular thickness with focal areas of ulceration. Neutrophilic infiltrate was seen in the superficial zone of ulcerated epithelium with fibrinous membrane. Underlying connective tissue was edematous and densely inflamed with diffuse inflammatory infiltrate predominantly lymphocytes, plasma cells, and neutrophils. Numerous

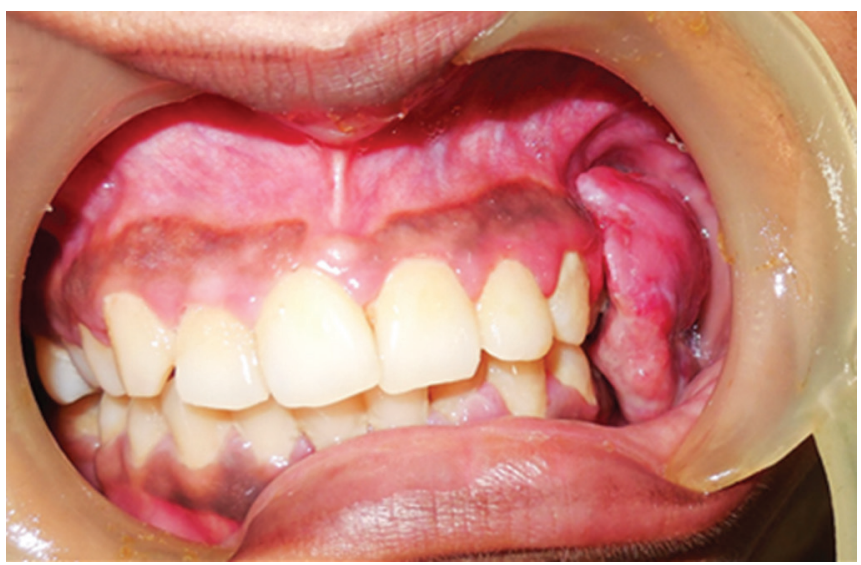

Fig. 2: Preoperative (frontal view)

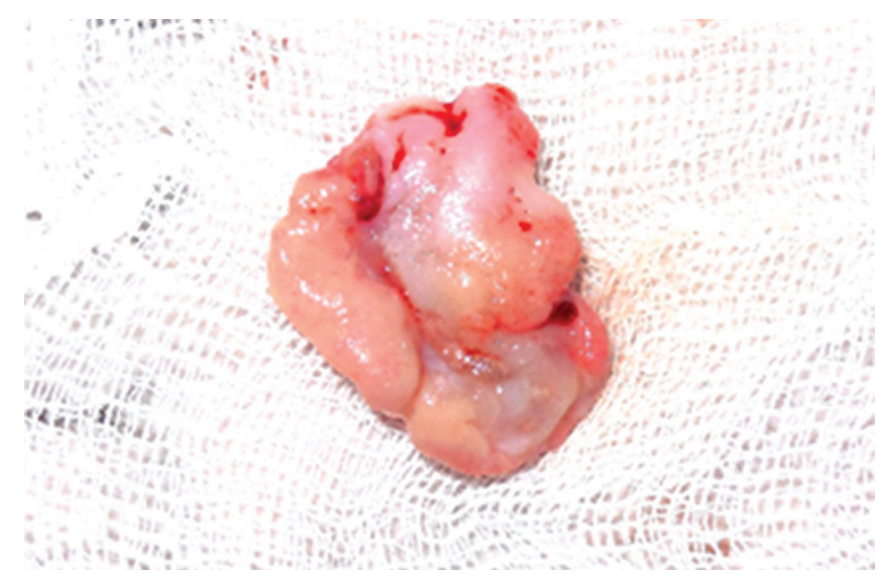

Fig. 4: Excised tissue (external surface) 


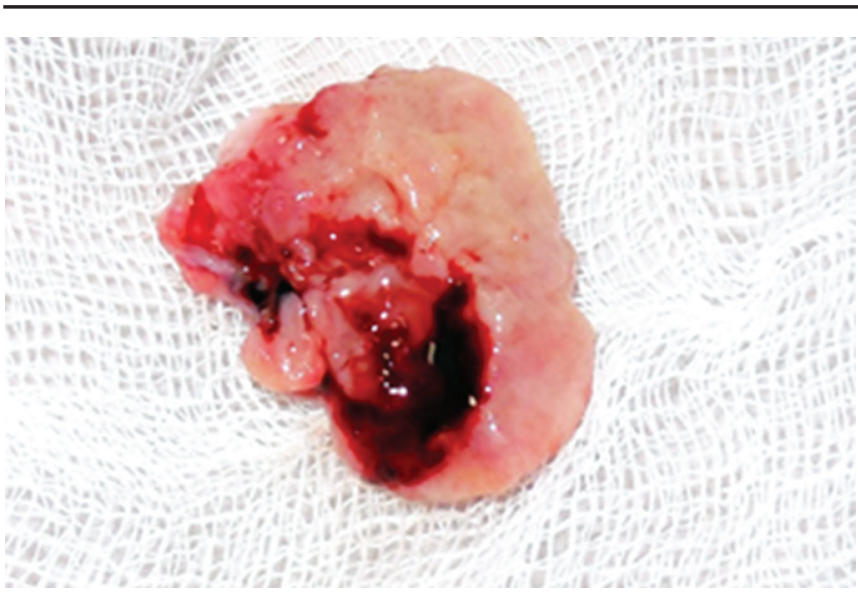

Fig. 5: Excised tissue (internal surface)

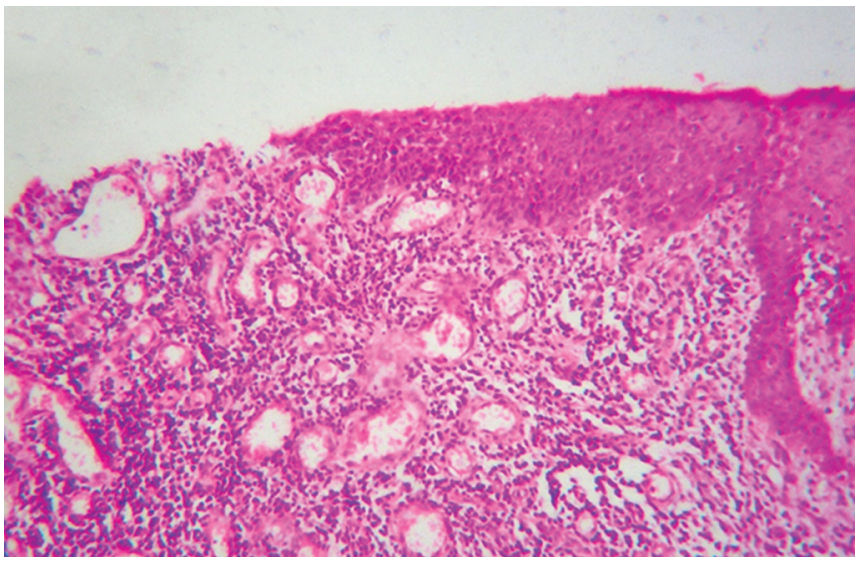

Fig. 7: Histological view

budding capillaries lined by plump endothelial cells with few vessels showing endothelial cell proliferation were seen. Few blood vessels were congested and showed thick walls. Focal areas of fibrosis and hyalinization and areas of hemorrhage and bacterial colonies were also seen. Thus, the clinical and histopathological features were diagnostic of pyogenic granuloma.

Healing was uneventful at the recall visit. The patient was reinstructed for maintenance of oral hygiene and a regular follow-up. No recurrence has been observed after 8 months of follow-up (Fig. 8).

\section{DISCUSSION}

The pyogenic granuloma or granuloma pyogenicum which appears during pregnancy is called pregnancy tumor. Some investigators consider pyogenic granuloma as a "reactive" or "reparative" tumor process. Regezi et al suggest that pyogenic granuloma represents an exuberant connective tissue proliferation to a known stimulus or injury-like calculus or foreign material within the gingival crevice. ${ }^{5}$

Several "etiologic factors" such as trauma, injury to a primary tooth, chronic irritation, hormones, drugs, gingival inflammation, preexisting vascular lesions, chronic

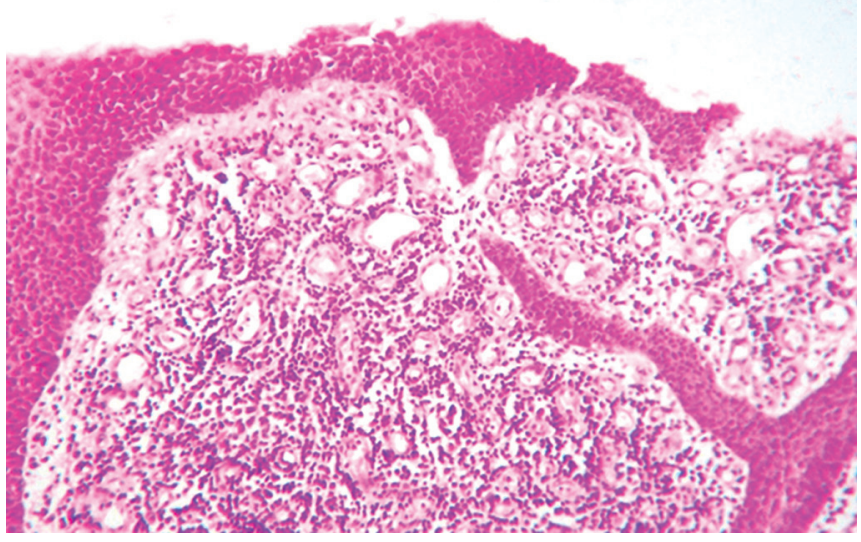

Fig. 6: Histological view

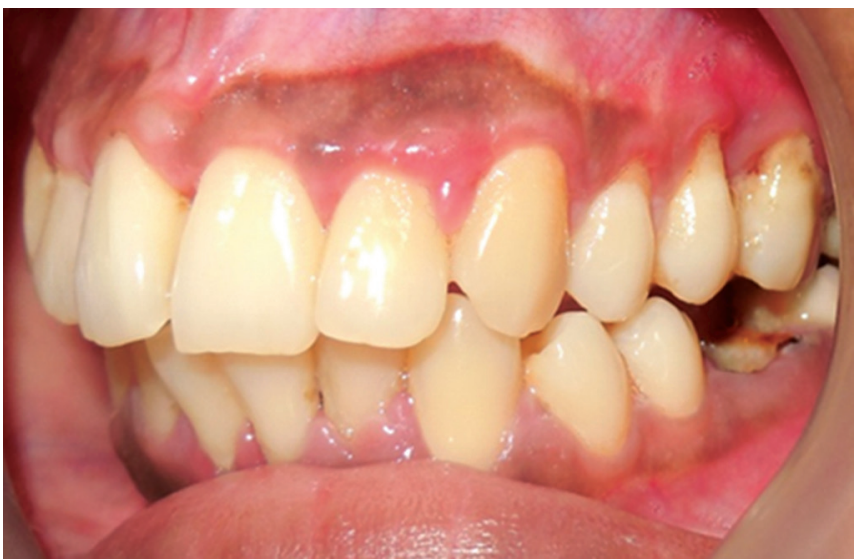

Fig. 8: Eight months postoperative view

irritation due to exfoliation of primary teeth, eruption of permanent teeth, defective fillings in the region of tumor, food impaction, periodontitis, toothbrush trauma, etc., have been suggested in the development of pyogenic granuloma. ${ }^{5}$ In the present case, the hormonal changes associated with pregnancy exaggerated the response to plaque.

Pregnancy is accompanied by remarkable endocrine alterations, both progesterone and estrogen are elevated due to continuous production of these hormones by the corpus luteum. These hormonal changes induce changes in vascular permeability, leading to gingival edema and an increased inflammatory response to bacterial plaque. ${ }^{4}$

Pregnancy itself cannot cause gingivitis, and gingivitis in pregnancy is caused by bacterial-hormonal interactions which may change the composition of plaque and thereby lead to gingival inflammation. Kornman and Loesche in 1980 reported that the subgingival flora changes to more anaerobic flora as pregnancy progresses with high predominance of Prevotella intermedia. This increase appears to be associated with elevations in systemic levels of estradiol and progesterone, which can substitute for menadion (vitamin K), an essential growth factor for Prevotella intermedia. ${ }^{6}$ 
During pregnancy, 0.2 to $9.6 \%$ of pregnant women experience localized gingival enlargement consistent with pyogenic granuloma. Clinically pyogenic granuloma is generally seen as a smooth or lobulated exophytic lesion with a pedunculated or a sessile base (Kostantinides A 2003). ${ }^{4}$ It rarely reaches more than $2 \mathrm{~cm}$ in size; however, in this case the lesion had enlarged to an extent that it was interfering with occlusion $(3 \times 2 \mathrm{~cm})$. Jafarzadeh et $\mathrm{al}^{7}$ stated that pyogenic granuloma shows a striking predilection for the gingiva, with interdental papillae being the most common site in $70 \%$ of the cases and more common in the maxillary anterior region.

In 1946 Ziskin and Ness compiled a clinical classification of pregnancy gingivitis as follows:

Class I: Characterized by bleeding gingiva with more or less, no other manifestations.

Class II: Characterized by changes in the interdental papilla-edema and swelling with subsequent blunting of interdental papilla.

Class III: Characterized by involvement of the free gum margin, which takes on the color and general appearance of a raspberry.

Class IV: Generalized hypertrophic gingivitis of pregnancy.

Class V: The pregnancy tumor. ${ }^{6}$

Thus, based on the above clinical classification of pregnancy gingivitis, the present case was classified as class V.

The consistency of the tumor gets firmer both with aging of the lesion and elimination of its etiological factors. Its color ranges from pink to red to purple, depending on the age of the lesion. Young pyogenic granulomas are highly vascular in appearance because they are composed predominantly of hyperplastic granulation tissue in which capillaries are prominent. Thus minor trauma to the lesion may cause considerable bleeding, due to its pronounced vascularity, whereas older lesions tend to become more collagenized and pink. ${ }^{7}$ In the present case during the time of excision the lesion was pinkish red in color.

Pyogenic granuloma may be confused with other benign and malignant conditions because of its appearance and evolution of growth; hence, biopsy findings are important in establishing diagnosis. Histologically, pyogenic granulomas are classified as the lobular capillary hemangioma type (LCH type) and the non-LCH type. The LCH type has proliferating blood vessels organized in lobular aggregates, no specific changes, such as edema, capillary dilation, or inflammatory granulation tissue reaction. The lobular area of the $\mathrm{LCH}$ type has a greater number of blood vessels with small luminal diameter than that in a non-LCH type of pyogenic granuloma. ${ }^{5,7}$
The non-LCH type consists of a vascular core resembling granulation tissue with foci of fibrous tissue. In the central area, a greater number of vessels with perivascular mesenchymal cells non-reactive for alpha smooth muscle actin (SMA) is detected as compared with the lobular area of the LCH type pyogenic granuloma. ${ }^{7}$ Thus, based on the histopathological features of the present case, it can be considered as non-LCH type of pyogenic granuloma since it shows a close resemblance of the histological features like edema and numerous budding capillaries lined by plump endothelial cells.

Spontaneous reduction in the size of enlarged lesion typically follows the termination of pregnancy. In the present case, even after parturition the enlargement showed no regression in size, so the lesion was surgically excised. A recurrence rate of $16 \%$ of lesions has been reported in the lesions after excision. Recurrence is believed to result from incomplete excision, failure to remove etiologic factors, or re-injury of the area. Also gingival lesions show a much higher recurrence rate than lesions from other oral mucosal sites. ${ }^{4}$ The patient is on regular maintenance visits since 8 months of treatment and no recurrence was seen.

\section{CONCLUSION}

Pregnancy tumor is a lesion mostly occurring on the gingiva in pregnancy. This case report presents a case of pregnancy tumor where in spite of its size and interference with occlusion patient did not give consent for the excision until parturition. This shows the lack of awareness among the pregnant patients about the oral health care during pregnancy. It is the responsibility of the dentist, gynecologist, and the periodontist to improve the oral health care awareness among such patients, motivating them for regular recall visits which will reduce the occurrence of pyogenic granuloma and other pregnancyrelated complications.

\section{ACKNOWLEDGMENT}

Authors would like to acknowledge Dr. Reyazullah, Reader, Department of Oral and Maxillofacial Surgery, VS Dental College and Hospital (VSDCH), Bengaluru for his support and guidance throughout the study.

\section{REFERENCES}

1. Yuan K, Jin YT, Lin MT. The detection and comparison of angiogenesis-associated factors in pyogenic granuloma by immunohistochemistry. J Periodontol 2000 May;71(5): 701-709.

2. Martins-Filho PRS, Piva MR, Da Silva LC, Reinheimer DM, Santos TS. Aggressive pregnancy tumor (pyogenic 
granuloma) with extensive alveolar bone loss mimicking a malignant tumor: case report and review of literature. Int J Morphol 2011;29(1):164-167.

3. Kurian B, Sasirekha E. Pyogenic granuloma - A case report and review. Int J Dent Sci Res 2014 Jun;2(3):66-68.

4. Guncu GN, Tozum TF, Caglayan F. Effects of endogenous sex hormones on the periodontium - Review of literature. Aust Dent J 2005 Sep;50(3):138-145.
5. Gomes SR, Shakir QJ, Thaker PV, Tavadia JK. Pyogenic granuloma of the gingiva: A misnomer? - A case report and review of literature. J Indian Soc Periodontol 2013 Jul-Aug;17(4):514-519.

6. Priya K, Sekar B, Augustine D, Murali S. Persistent pregnancy tumor: a case report with review of literature. Oral Maxillofacial Pathol J 2012 Jul-Dec;3(2):264-268.

7. Jafarzadeh H, Sanatkhani M, Mohtasham N. Oral pyogenic granuloma: a review. J Oral Sci 2006 Dec;48(4):167-175. 